\title{
Some New Properties in Fredholm Theory, Schechter Essential Spectrum, and Application to Transport Theory
}

\author{
Boulbeba Abdelmoumen, ${ }^{1}$ Abdelkader Dehici, ${ }^{2}$ Aref Jeribi, ${ }^{1}$ and Maher Mnif ${ }^{1}$ \\ ${ }^{1}$ Department of Mathematics, Faculty of Science of Sfax, Sfax 3018, Tunisia \\ ${ }^{2}$ Département des Sciences Exactes, Université 8 Mai 1945, BP 401, Guelma 24000, Algeria \\ Correspondence should be addressed to Aref Jeribi, aref.jeribi@fss.rnu.tn
}

Received 19 April 2007; Revised 11 July 2007; Accepted 24 September 2007

Recommended by Nikolaos S. Papageorgiou

The theory of measures of noncompactness has many applications on topology, functional analysis, and operator theory. In this paper, we consider one axiomatic approach to this notion which includes the most important classical definitions. We give some results concerning a certain class of semi-Fredholm and Fredholm operators via the concept of measures of noncompactness. Moreover, we establish a fine description of the Schechter essential spectrum of closed densely defined operators. These results are exploited to investigate the Schechter essential spectrum of a multidimensional neutron transport operator.

Copyright ( 12008 Boulbeba Abdelmoumen et al. This is an open access article distributed under the Creative Commons Attribution License, which permits unrestricted use, distribution, and reproduction in any medium, provided the original work is properly cited.

\section{Introduction}

Let $(X,\|\cdot\|)$ be an infinite-dimensional Banach space. The open ball of $X$ will be denoted by $B_{X}$ and its closure by $\bar{B}_{X}$. We denote by $\mathcal{C}(X)$ (resp., $\mathcal{L}(X)$ ) the set of all closed densely defined linear operators (resp., the space of all bounded linear operators) on $X$. The set of all compact operators of $\mathcal{L}(X)$ is designed by $\mathcal{K}(X)$. Let $T \in \mathcal{C}(X)$, we write $\mathcal{N}(T) \subseteq X$ for the null space and $\mathcal{R}(T) \subseteq X$ for the range of $T$. We set $\alpha(T):=\operatorname{dim} \mathcal{N}(T)$ and $\beta(T):=\operatorname{codim} \mathcal{R}(T)$. The set of upper semi-Fredholm operators is defined by

$$
\Phi_{+}(X)=\{T \in \mathcal{C}(X) \text { such that } \alpha(T)<\infty, \mathcal{R}(T) \text { closed in } X\},
$$

and the set of lower semi-Fredholm operators is defined by

$$
\Phi_{-}(X)=\{T \in \mathcal{C}(X) \text { such that } \beta(T)<\infty(\text { then } \mathcal{R}(T) \text { closed in } X)\} \text {. }
$$


$\Phi(X):=\Phi_{+}(X) \cap \Phi_{-}(X)$ is the set of Fredholm operators in $\mathcal{C}(X)$, while $\Phi_{ \pm}(X):=\Phi_{+}(X) \cup \Phi_{-}(X)$ is the set of semi-Fredholm operators in $\mathcal{C}(X)$. If $T \in \Phi(X)$, the number $i(T):=\alpha(T)-\beta(T)$ is called the index of $T$. The spectrum of $T$ will be denoted by $\sigma(T)$. The resolvent set of $T, \rho(T)$, is the complement of $\sigma(T)$ in the complex plane. A complex number $\lambda$ is in $\Phi_{+T}, \Phi_{-T}, \Phi_{ \pm T}$, or $\Phi_{T}$ if $\mathcal{\lambda}-T$ is in $\Phi_{+}(X), \Phi_{-}(X), \Phi_{ \pm}(X)$, or $\Phi(X)$, respectively. In the next proposition we recall some well-known properties of those sets (see, e.g., $[11,16,30])$.

Proposition 1.1. For any $T \in \mathcal{C}(X)$,

(i) $\Phi_{+T}, \Phi_{-T}$ and $\Phi_{T}$ are open,

(ii) $i(\lambda-T)$ is constant on any component of $\Phi_{T}$.

There are many ways to define the essential spectrum of a closed densely defined linear operator on a Banach space. Hence several definitions of the essential spectrum may be found in the literature; see, for example, [16] or the comments in [30, Chapter 11, page 283]. Various notions of essential spectrum appear in the applications of spectral theory (see, e.g., [13, 16, 21]). Throughout this paper we are concerned with the so-called Schechter essential spectrum.

Definition 1.2. Let $T \in \mathcal{C}(X)$. Define the Schechter essential spectrum of the operator $T$ by

$$
\sigma_{\mathrm{ess}}(T)=\bigcap_{K \in \mathcal{K}(X)} \sigma(T+K) .
$$

The following proposition gives a characterization of the Schechter essential spectrum by means of Fredholm operators.

Proposition 1.3 (see [30, Theorem 5.4, page 180]). Let $T \in \mathcal{C}(X)$. Then

$$
\lambda \notin \sigma_{\text {ess }}(T) \quad \text { iff } \lambda \in \Phi_{T}^{0}
$$

where $\Phi_{T}^{0}:=\left\{\lambda \in \Phi_{T}\right.$ such that $\left.i(\lambda-T)=0\right\}$.

Definition 1.4. An operator $T \in \mathcal{L}(X)$ is said to be weakly compact if $T(B)$ is relatively weakly compact for every bounded subset $B \subset X$.

The family of weakly compact operators on $X, \mathcal{W}(X)$, is a closed two-sided ideal of $\mathcal{L}(X)$ containing $\mathcal{K}(X)$ (see $[8,12])$.

Definition 1.5. A Banach space $X$ is said to have the Dunford-Pettis property (for short property DP) if for each Banach space $Y$ every weakly compact operator $T: X \rightarrow Y$ takes weakly compact sets in $X$ into norm compact sets of $Y$.

It is well known that any $L_{1}$-space has the property DP [9]. Also if $\Omega$ is a compact Hausdorff space, $C(\Omega)$ has the property DP [15]. For further examples we refer to [5] or [8, pages $494,497,508$, and 511]. Note that the property DP is not preserved under conjugation. However, if $X$ is a Banach space whose dual has the property DP, then $X$ has the property DP (see, e.g., [15]). Furthermore, if the Banach space $X$ has the property DP, then $\mathcal{W}(X) \mathcal{W}(X) \subset \mathcal{K}(X)$, where $\mathcal{W}(X) \mathcal{W}(X)=\{J K: J, K \in \mathcal{W}(X)\}$ (see [18, Lemma 2.1]). For more information we refer to the paper by Diestel [5] which contains a survey and exposition of the Dunford-Pettis property and related topics. 
One of the central questions in the study of the Schechter essential spectrum of closed densely defined linear operators on Banach spaces $X$ consists of showing what are the conditions that we must impose on $K \in \mathcal{L}(X)$ such that, for $T \in \mathcal{C}(X), \sigma_{\text {ess }}(T+K)=$ $\sigma_{\text {ess }}(T)$. If $K$ is a compact operator on Banach spaces, then $\sigma_{\text {ess }}(T+K)=\sigma_{\text {ess }}(T)$ (see Definition 1.2). If $K$ is a strictly singular on $L_{p}$-spaces, then $\sigma_{\text {ess }}(T+K)=\sigma_{\text {ess }}(T)$ (see [25, Theorem 3.2]). If $K$ is weakly compact on Banach spaces which possess the DunfordPettis property, then $\sigma_{\text {ess }}(T+K)=\sigma_{\text {ess }}(T)$ (see [23, Theorem 3.2]). If $K \in \mathcal{L}(X)$ and $(\lambda-T)^{-1} K$ is strictly singular (resp., weakly compact) on $L_{p}$-spaces $p>1$ (resp., on Banach spaces which possess the Dunford-Pettis property), then $\sigma_{\text {ess }}(T+K)=\sigma_{\text {ess }}(T$ ) (see $[17,18])$. In [19], Jeribi extended this analysis of the Schechter essential spectrum to the case of general Banach spaces and he proves that $\sigma_{\text {ess }}(T+K)=\sigma_{\text {ess }}(T)$ for all $K \in \mathcal{L}(X)$ such that $(\lambda-T)^{-1} K \in \mathcal{J}(X)$, where $\supset(X)$ is an arbitrary two-sided ideal of $\mathcal{L}(X)$ satisfying $\mathcal{K}(X) \subset \supset(X) \subset \mathcal{F}(X)$, where $\mathcal{F}(X)=\{F \in \mathcal{L}(X)$ such that $F+U \in \Phi(X)$ whenever $U \in \Phi(X)\}$. Recently, in [20], the Schechter essential spectrum is characterized by

$$
\sigma_{\mathrm{ess}}(T)=\bigcap_{K \in \mathcal{M}_{n}(X)} \sigma(T+K),
$$

where $T \in \mathcal{C}(X)$ and $\mathcal{M}_{n}(X):=\left\{A \in \mathcal{L}(X):(A B)^{n} \in \mathcal{K}(X), \forall B \in \mathcal{L}(X)\right\}$. In our paper, using the concept of measure of noncompactness, we show in Theorem 3.1 (see Section 3 ) that, for $P$ and $Q$ two complex polynomials such that $Q$ divides $P-1$ and $\gamma$ is the Kuratowski measure of noncompactness, we have

(i) if $\gamma(P(T))<1$, then $Q(T) \in \Phi_{+}(X)$,

(ii) if $\gamma(P(T))<1 / 2$, then $Q(T) \in \Phi(X)$.

We apply this result to give a new characterization of the Schechter essential spectrum (see Theorem 3.5) by means of the measure of noncompactness and we give sufficient conditions on the perturbed operator (see Corollary 4.12) to have the invariance of the Schechter essential spectrum on Banach space which possesses the Dunford-Pettis property. More precisely, we show that in (1.5), the set $\mathcal{M}_{n}(X)$ can be replaced by the more general class:

$$
\mathcal{G}_{T}^{n}(X)=\left\{K \in \mathcal{L}(X): \gamma\left(\left[(\lambda-T-K)^{-1} K\right]^{n}\right)<\frac{1}{2} \forall \lambda \in \rho(T+K)\right\}
$$

(see Theorem 3.5), and we prove that for $X$ having the property DP and $T \in \mathcal{C}(X)$, $\sigma_{\text {ess }}(T+K)=\sigma_{\text {ess }}(T)$, for all $K$ in a subgroup of $\mathcal{G}_{T}^{n}(X)$.

Finally, we apply the obtained results to study the Schechter essential spectrum of the multidimensional neutron transport equation which governs the time evolution of the distribution of neutrons in a nuclear reactor (cf. [7, 14, 22, 32]). In [24], it was shown that if $K$ is a regular collusion operator, then the Schechter essential spectrum of one-dimensional transport operator with general boundary conditions on $L_{1}$ spaces is given as $\sigma_{\text {ess }}(T+K)=\{\lambda \in$ $\mathbb{C}$ such that $\left.\operatorname{Re} \lambda \leq-\lambda^{*}\right\}$, where $T$ is the streaming operator and $\lambda^{*}:=\lim \inf _{|\xi| \rightarrow 0} \sigma(\xi)$. The possibility of the above result is due to the fact that, in slab geometry, if $K$ is regular, then $(\lambda-T)^{-1} K$ is weakly compact (cf. [24, Proposition 3.2(i)]). Unfortunately, for multidimensional neutron transport equation, $(\lambda-T)^{-1} K$ is not compact nor weakly compact. For this reason, in [26], the authors have shown only the following inclusion $\sigma_{\text {ess }}(T+K) \subset\left\{\lambda \in \mathbb{C}\right.$ such that $\left.\operatorname{Re} \lambda \leq-\lambda^{*}\right\}$ 
(see Theorem 5.3). In this paper, we give sufficient conditions to replace the above inclusion by equality (see Theorem 5.3).

Our paper is organized as follows. In Section 2, we consider one axiomatic approach to the notion of measure of noncompactness. In Section 3, we use the notion of measure of noncompactness to establish some results concerning the class of Fredholm operators and to apply the obtained results to give a new characterization of the Schechter essential spectrum. The main result of this section is Theorem 3.5. In Section 4, we prove that under some conditions on the perturbed operator, we get the invariance of the Schechter essential spectrum on a Banach space which possesses the Dunford-Pettis property (see Corollary 4.12). Finally, in Section 5, we apply the result of Theorem 4.11 to investigate the Schechter essential spectrum of the multidimensional neutron transport equation.

\section{Measure of noncompactness}

The notion of measure of noncompactness turned out to be a useful tool in some problems of topology, functional analysis, and operator theory (see [1, 3, 6, 27, 29]). In order to recall the measure of noncompactness, let $(X,\|\cdot\|)$ be an infinite-dimensional Banach space. The open ball of $X$ will be denoted by $B_{X}$ and its closure by $\bar{B}_{X}$. We denote by $M_{X}$ the family of all nonempty and bounded subsets of $X$ while $N_{X}$ denotes its subfamily consisting of all relatively compact sets. Moreover, let us denote by $\operatorname{conv}(A)$ the convex hull of a set $A \subset X$.

Let us recall the following definition.

Definition 2.1 (see [3]). A mapping $\mu: M_{X} \rightarrow[0,+\infty$ [ is said to be a measure of noncompactness in the space $X$ if it satisfies the following conditions:

(i) the family $\operatorname{Ker}(\mu):=\left\{D \in M_{X}: \mu(D)=0\right\}$ is nonempty and $\operatorname{Ker}(\mu) \subset N_{X}$,

for $A, B \in M_{X}$, we have the following:

(ii) if $A \subset B$, then $\mu(A) \leq \mu(B)$,

(iii) $\mu(\bar{A})=\mu(A)$,

(iv) $\mu(\overline{\operatorname{conv}(A)})=\mu(A)$,

(v) $\mu(\lambda A+(1-\lambda) B) \leq \lambda \mu(A)+(1-\lambda) \mu(B)$, for all $\lambda \in[0,1]$,

(vi) if $\left(A_{n}\right)_{n \in \mathbb{N}}$ is a sequence of sets from $M_{X}$ such that $A_{n+1} \subset A_{n}, \bar{A}_{n}=A_{n}(n=1,2, \ldots)$ and $\lim _{n \rightarrow+\infty} \mu\left(A_{n}\right)=0$, then $A_{\infty}=\bigcap_{n=1}^{\infty} A_{n}$ is nonempty and $A_{\infty} \in \operatorname{Ker}(\mu)$.

The family $\operatorname{Ker}(\mu)$ described in Definition 2.1(i) is called the kernel of the measure of noncompactness $\mu$.

Definition 2.2. A measure of noncompactness $\mu$ is said to be sublinear if for all $A, B \in M_{X}$, it satisfies the following two conditions:

(i) $\mu(\lambda A)=|\lambda| \mu(A)$ for $\lambda \in \mathbb{R}$ ( $\mu$ is said to be homogenous),

(ii) $\mu(A+B) \leq \mu(A)+\mu(B)$ ( $\mu$ is said to be subadditive).

Definition 2.3. A measure of noncompactness $\mu$ is referred to as measure with maximum property if $\max (\mu(A), \mu(B))=\mu(A \cup B)$. 
Definition 2.4. A measure of noncompactness $\mu$ is said to be regular if $\operatorname{Ker}(\mu)=N_{X}$, sublinear, and has maximum property. are

For $A \in M_{X}$, the most important examples of measures of noncompactness (see [27])

(i) Kuratowski measure of noncompactness

$$
\gamma(A)=\inf \{\varepsilon>0: A \text { may be covered by finitely many sets of diameter } \leq \varepsilon\},
$$

(ii) Hausdorff measure of noncompactness

$\bar{\gamma}(A)=\inf \{\varepsilon>0: A$ may be covered by finitely many open balls of radius $\leq \varepsilon\}$.

Note that these measures $\gamma$ and $\bar{\gamma}$ are regular. The relations between these measures are given by the following inequalities, which are obtained by Daneš [4]:

$$
\bar{\gamma}(A) \leq \gamma(A) \leq 2 \bar{\gamma}(A), \quad \text { for any } A \in M_{X} .
$$

Let $T \in \mathcal{L}(X)$. We say that $T$ is $k$-set-contraction if for every set $A \in M_{X}$, we have $\gamma(T(A))$ $\leq k \gamma(A) . T$ is called $k$-ball-contraction if $\bar{\gamma}(T(A)) \leq k \bar{\gamma}(A)$ for every set $A \in M_{X}$. We define $\gamma(T)$ and $\bar{\gamma}(T)$, respectively, by

$$
\begin{aligned}
& \gamma(T):=\inf \{k: T \text { is } k \text {-set-contraction }\}, \\
& \bar{\gamma}(T):=\inf \{k: T \text { is } k \text {-ball-contraction }\} .
\end{aligned}
$$

In the following lemma, we give some important properties of $\gamma(T)$ and $\bar{\gamma}(T)$.

Lemma 2.5 (see $[2,10])$. Let $X$ be a Banach space and $T \in \mathcal{L}(X)$.

(i) $(1 / 2) \gamma(T) \leq \bar{\gamma}(T) \leq 2 \gamma(T)$.

(ii) $\gamma(T)=0$ if and only if $\bar{\gamma}(T)=0$ if and only if $T$ is compact.

(iii) If $T, S \in \mathcal{L}(X)$, then $\gamma(S T) \leq \gamma(S) \gamma(T)$ and $\bar{\gamma}(S T) \leq \bar{\gamma}(S) \bar{\gamma}(T)$.

(iv) If $K \in \mathcal{K}(X)$, then $\gamma(T+K)=\gamma(T)$ and $\bar{\gamma}(T+K)=\bar{\gamma}(T)$.

(v) $\gamma\left(T^{*}\right) \leq \bar{\gamma}(T)$ and $\gamma(T) \leq \bar{\gamma}\left(T^{*}\right)$, where $T^{*}$ denotes the dual operator of $T$.

\section{A characterization of the Schechter essential spectrum}

Let $X$ be a Banach space. The open ball of $X$ will be denoted by $B_{X}$ and its closure by $\bar{B}_{X}$. We start our investigation with the following useful result.

Theorem 3.1. Let $X$ be a Banach space, $T \in \mathcal{L}(X)$, and $P, Q$ two complex polynomials satisfying $Q$ which divides $P-1$.

(i) If $\gamma(P(T))<1$, then $Q(T) \in \Phi_{+}(X)$.

(ii) If $\gamma(P(T))<1 / 2$, then $Q(T) \in \Phi(X)$.

To prove this theorem the following lemma is required. 
Lemma 3.2. Assume that the hypotheses of Theorem 3.1 hold true. Let $M \subset X$ and let $A=\left\{x \in \bar{B}_{X}\right.$ : $Q(T)(x) \in M\}$. If $M$ is compact and $\gamma(P(T))<1$, then $A$ is compact or empty.

Proof. Assume that $A$ is not empty. According to the hypothesis $Q$ divides $P-1$, there exists $H$, a complex polynomial such that $P=H Q+1$. Consider $x \in A$ and $z \in M$ such that $Q(T)(x)=z$, then we get $H(T) Q(T)(x)+x=H(T)(z)+x$, which implies $x=P(T) x-H(T)(z)$. Since a continuous image of a compact set by a continuous operator is also compact, it follows that

$$
\widetilde{A}=\{-H(T)(z): z \in M\}
$$

is compact as well. Obviously, $A \subset P(T) A+\widetilde{A}$, so using the regularity of $\gamma$, we get

$$
\gamma(A) \leq \gamma(P(T) A)+\gamma(\tilde{A}) \leq \gamma(A) \gamma(P(T)) \text {. }
$$

Since $\gamma(P(T))<1$, then $\gamma(A)=0$. Consequently, by Definition 2.1 and the fact that $A$ is closed, we infer that $A$ is compact.

Proof of Theorem 3.1. (i) First we prove that $\alpha(Q(T))<\infty$. To do so, it suffices to establish that the set $\mathcal{N}(Q(T)) \cap \bar{B}_{X}$ is compact, where $\mathcal{N}(Q(T))$ and $\bar{B}_{X}$ denote, respectively, the null space of the operator $Q(T)$ and the closed unit ball of $X$. The result follows from Lemma 3.2 with $M=\{0\}$.

In order to complete the proof of (i), we will check that $\mathcal{R}(Q(T)$ ) (the range of $Q(T)$ ) is closed. Indeed, since $\mathcal{N}(Q(T))$ is finite dimensional, then there exists a closed infinitedimensional subspace $Y$ in $X$ such that $X=\mathcal{N}(Q(T)) \oplus Y$.

We claim that there exists $\delta>0$ satisfying $\delta\|Q(T)(x)\| \geq\|x\|$ for every $x \in Y$. Assume the contrary, for every $n \in \mathbb{N}$, there exists $x_{n} \in Y$ satisfying $\left\|x_{n}\right\|=1$ and $\left\|Q(T)\left(x_{n}\right)\right\| \leq 1 / n$. Hence $Q(T)\left(x_{n}\right) \rightarrow 0$ (when $n \rightarrow+\infty$ ). It follows from Lemma 3.2 with $M=\left\{Q(T)\left(x_{n}\right): n \in \mathbb{N}\right\} \cup\{0\}$ that the sequence $\left(x_{n}\right)_{n \in \mathbb{N}}$ admits a subsequence $\left(x_{n_{k}}\right)_{k \in \mathbb{N}}$ which converges to $x_{0} \in Y$. Clearly, $\left\|x_{0}\right\|=1$ and $Q(T)\left(x_{0}\right)=0$. This is a contradiction. This proves the claim.

Using [31], it is easy to conclude that $R(Q(T))$ is closed. This ends the proof of (i).

(ii) Assume that $\gamma(P(T))<1 / 2$. Combining the assertions (i) and (v) of Lemma 2.5 one has $\gamma\left(P(T)^{*}\right) \leq 2 \gamma(P(T))<1$, where $P(T)^{*}$ stands for the dual of the operator $P(T)$. Arguing as in the proof of (i), we get $\alpha\left(Q(T)^{*}\right)=\beta(Q(T))<\infty$. This completes the proof of the theorem.

As a consequence of Theorem 3.1 we have the following corollary.

Corollary 3.3. Let $X$ be a Banach space, $T \in \mathcal{L}(X)$, and let $P$ be a complex polynomial nonconstant satisfying $P(0)=1$.

(i) If $\gamma(P(T))<1$, then $T \in \Phi_{+}(X)$.

(ii) If $\gamma(P(T))<1 / 2$, then $T \in \Phi(X)$.

(iii) If $\gamma(I+T)<1$, then $T \in \Phi(X)$.

Proof. (i)-(ii) Since $P(0)=1$, then $Q(z):=z$ divides $(P(z)-1)$ and the result follows from Theorem 3.1.

(iii) If $\gamma(I+T)<1$, then $\lim _{k \rightarrow+\infty}(\gamma(I+T))^{k}=0$. So, there exists $k_{0} \in \mathbb{N}^{*}$ such that $(\gamma(I+T))^{k_{0}} \leq 1 / 2$. Using Lemma 2.5(iii), we deduce that $\gamma\left((I+T)^{k_{0}}\right) \leq 1 / 2$. So, the result is consequence immediate from (ii) with $P(z):=(1+z)^{k_{0}}$ and $Q(z):=z$. 
Corollary 3.4. Let $X$ be a Banach space and $T \in \mathcal{L}(X)$.

If $\gamma\left(T^{m}\right)<1$, for some $m>0$, then $(I-T)$ is a Fredholm operator with $i(I-T)=0$.

Proof. If $\gamma\left(T^{m}\right)<1$, then $\lim _{k \rightarrow+\infty}\left(\gamma\left(T^{m}\right)\right)^{k}=0$. Arguing as in the proof of Corollary 3.3(iii), there exists $k_{0} \in \mathbb{N}^{*}$ such that $\gamma\left(T^{m k_{0}}\right) \leq 1 / 2$. So, applying Theorem 3.1 with $P(z):=z^{m k_{0}}$ and $Q(z):=1-z$ we conclude that $Q(T):=(I-T) \in \Phi(X)$. Next, note that for $t \in[0,1]$, we have $\gamma\left((t T)^{m k_{0}}\right)<1 / 2$ and therefore $(I-t T)$ is a Fredholm operator on $X$. On the other hand, the fact that the index is constant on any component of $\Phi(X)$ (see Proposition 1.1) and the compactness of $[0,1]$ imply that $i(Q(T))=i(I-t T)=i(I)=0$.

In what follows, we will give a refinement of the definition of the Schechter essential spectrum. For this, let $X$ be a Banach space and let $n \in \mathbb{N}^{*}$. For each $T \in \mathcal{C}(X)$, we denote

$$
\sigma_{W}^{n}(T)=\bigcap_{K \in \mathcal{C}_{T}^{n}(X)} \sigma(T+K)
$$

where $\mathcal{G}_{T}^{n}(X)=\left\{K \in \mathcal{L}(X): \gamma\left(\left[(\lambda-T-K)^{-1} K\right]^{n}\right)<1 / 2 \forall \lambda \in Q(T+K)\right\}$.

The main result of this section is the following theorem.

Theorem 3.5. For each $T \in \mathcal{C}(X)$,

$$
\sigma_{\mathrm{ess}}(T)=\sigma_{W}^{n}(T)
$$

Proof. We first claim that $\sigma_{\text {ess }}(T) \subset \sigma_{W}^{n}(T)$. Indeed, if $\lambda \notin \sigma_{W}^{n}(T)$, then there exists $K \in \mathcal{G}_{T}^{n}(X)$ such that $\lambda \in \rho(T+K)$. So, $\lambda \in \rho(T+K)$ and $\gamma\left(\left[(\lambda-T-K)^{-1} K\right]^{n}\right)<1 / 2$. Hence, applying Corollary 3.4(i), we get

$$
\left[I+(\lambda-T-K)^{-1} K\right] \in \Phi(X), \quad i\left[I+(\lambda-T-K)^{-1} K\right]=0 .
$$

Moreover, we have

$$
\lambda-T=(\lambda-T-K)\left[I+(\lambda-T-K)^{-1} K\right]
$$

then

$$
(\lambda-T) \in \Phi(X), \quad i(\lambda-T)=0 .
$$

Finally, the use of Proposition 1.3 shows that $\lambda \notin \sigma_{\text {ess }}(T)$ which proves our claim.

On the other hand, since $\mathcal{K}(X) \subset \mathcal{G}_{T}^{n}(X)$, we infer that $\sigma_{W}^{n}(T) \subset \sigma_{\text {ess }}(T)$ which completes the proof of the theorem.

Corollary 3.6. Let $n \in \mathbb{N}^{*}, T \in \mathcal{C}(X)$, and let $\mathscr{L}(X)$ be any subset of $\mathcal{L}(X)$ satisfying $\mathcal{K}(X) \subset$ $\mathscr{H}(X) \subset \mathcal{G}_{T}^{n}(X)$.Then $\sigma_{\mathrm{ess}}(T)=\bigcap_{K \in \mathscr{H}(X)} \sigma(T+K)$.

Proof. The fact is that $\mathscr{H}(X) \subset \mathcal{G}_{T}^{n}(X)$ then $\bigcap_{K \in \mathcal{G}_{T}^{n}(X)} \sigma(T+K) \subset \bigcap_{K \in \mathscr{H}(X)} \sigma(T+K)$. Using Theorem 3.5, we get $\sigma_{\text {ess }}(T) \subset \bigcap_{k \in \mathscr{d}(X)} \sigma(T+K)$. On the other hand, since $\mathcal{K}(X) \subset \mathscr{l}(X)$, we infer that $\bigcap_{k \in \mathscr{d}(X)} \sigma(T+K) \subset \sigma_{\text {ess }}(T)$ which completes the proof. 
Corollary 3.7. Let $T \in \mathcal{C}(X)$. Consider that $\supset_{T}(X)$ is included in $\mathcal{G}_{T}^{n}(X)$, containing the subspace of all compact operators $\mathcal{K}(X)$ and checking: for all $K, K^{\prime} \in \supset_{T}(X), K \pm K^{\prime} \in \supset_{T}(X)$. Then, for each $K \in \supset_{T}(X)$,

$$
\sigma_{\mathrm{ess}}(T)=\sigma_{\mathrm{ess}}(T+K)
$$

Proof. We denote that

$$
\sigma_{W}^{\prime}(T)=\bigcap_{K \in \supset_{T}(X)} \sigma(T+K)
$$

From Corollary 3.6, we have $\sigma_{\text {ess }}(T)=\sigma_{W}^{\prime}(T)$. Furthermore, for each $K \in \supset_{T}(X)$, we have $\supset_{T}(X)+K=\supset_{T}(X)$. Then $\sigma_{W}^{\prime}(T+K)=\sigma_{W}^{\prime}(T)$. Hence for each $K \in \supset_{T}(X)$, we get

$$
\sigma_{\mathrm{ess}}(T+K)=\sigma_{W}^{\prime}(T+K)=\sigma_{W}^{\prime}(T)=\sigma_{\mathrm{ess}}(T),
$$

which completes the proof.

\section{Invariance of the Schechter essential spectrum in Dunford-Pettis space}

In this section, we will establish the invariance of the Schechter essential spectrum in a Banach space $X$ which possesses the Dunford-Pettis property. In what follows, we will assume that $T \in \mathcal{C}(X)$ and satisfies the hypothesis $(\mathbb{A})$, that is,

(i) for all $R \in \mathcal{L}(X)$, there exist $\lambda \in \mathbb{R}$ such that $] \lambda,+\infty[\subset \rho(T+R)$,

(ii) $\rho_{\text {ess }}(T)$ is a connected set of $\mathbb{C}$.

Remark 4.1. Let $T \in \mathcal{C}(X)$. If $T$ generates a $C_{0}$-semigroup and $\rho_{\text {ess }}(T)$ is a connected set, then $T$ satisfies the hypothesis (A).

Definition 4.2. An operator $R \in \mathcal{L}(X)$ is called $T$-Regular if, for all $\lambda \in \rho(T), R(\lambda-T)^{-1} R$ is weakly compact and $\rho_{\text {ess }}(T+R)$ is a connected set of $\mathbb{C}$.

We note that $\mathcal{R}_{T}(X)$ is the set of all $T$-Regular operators. We start by giving some lemmas useful for the proof of the main result of this section.

Lemma 4.3. Assume that $R$ is $T$-Regular. Then, for all $\lambda \in \rho(T+R) \cap \rho(T), R(\lambda-T-R)^{-1} R$ is weakly compact.

Proof. The result follows from the resolvent identity:

$$
R(\lambda-T-R)^{-1} R-R(\lambda-T)^{-1} R=R(\lambda-T-R)^{-1} R(\lambda-T)^{-1} R .
$$

Remark 4.4. (i) If $R$ is $T$-Regular then, for all $\lambda \in Q(T+R) \cap \rho(T),\left[(\lambda-T-R)^{-1} R\right]^{4}$ is compact. (ii) If $\rho_{\text {ess }}(T)$ is a connected set of $\mathbb{C}$, then $\mathcal{K}(X) \subset \mathcal{R}_{T}(X)$.

Lemma 4.5. Let $\Omega$ be an open connected set of $\mathbb{C}$, let $Y$ be a Banach space, and let $f: \Omega \rightarrow \mathcal{L}(Y)$ be an analytic operator. 
Define $K(f)=\{\lambda \in \Omega$ such that $f(\lambda)$ is compact $\}$. Then one of the two possibilities must hold:

(a) $K(f)=\Omega$,

(b) $K(f)$ does not have a point of accumulation in $\Omega$.

Proof. Let $E=\{\lambda \in \Omega ; \lambda$ is point of accumulation of $K(f)$ in $\Omega\}$. If $\lambda \in E$, then there exists $\left(\lambda_{n}\right)_{n} \in K(f)$ such that $\lambda_{n}$ converges to $\lambda$. Since $f$ is continuous, then $f\left(\lambda_{n}\right)$ converges to $f(\lambda)$. As $f\left(\lambda_{n}\right)$ is compact, $f(\lambda)$ will be compact, so $E \subset K(f)$. We fix $\lambda \in K(f)$ and we choose $r>0$, such that $B(\lambda, r) \subset \Omega$. Since $f$ is analytic in $B(\lambda, r)$, then $f(z)=\sum_{n \geq 0} A_{n}(z-\lambda)^{n}$, where $\left(A_{n}\right)_{n}$ are bounded operators and independent of $z$. We have two possibilities.

(i) $A_{n}$ is compact for all $n \in \mathbb{N}$, then $B(\lambda, r) \subset K(f)$. So, each point $z \in B(\lambda, r)$ is an accumulation point of $K(f)$. We deduce that $B(\lambda, r) \subset E$ and $\lambda \in \stackrel{\circ}{E}$.

(ii) There exists a smaller integer $m$, such that $A_{m}$ is not compact. In this case, we write for $z \in B(\lambda, r)$,

$$
f(z)=\sum_{k=0}^{m-1} A_{k}(z-\lambda)^{k}+(z-\lambda)^{m} g(z),
$$

where $g(z)=\sum_{k=0}^{+\infty} A_{m+k}(z-\lambda)^{k}$. Furthermore $g(\lambda)$ is not compact, using the continuity of $g$, we get a neighborhood $V(\lambda)$ of $\lambda$ including in $B(\lambda, r)$ such that $g(\mu)$ is not compact for all $\mu \in V(\lambda)$. Indeed, suppose that for all $n>0$, there exists $\lambda_{n} \in B(\lambda, 1 / n)$ such that $g\left(\lambda_{n}\right)$ is compact. Since $\lim _{n \rightarrow+\infty} \lambda_{n}=\lambda$ and $g$ is continuous, then $g(\lambda)$ is compact, contradicting $g(\lambda)$ is not compact. So, $f(\mu)$ is not compact for all $\mu \in V(\lambda)$. Hence $\lambda$ is an isolated point of $K(f)$.

Let $\lambda \in E$, the first possibility holds, thus $E$ is open. Let $F=\Omega \backslash E$. It follows from the definition of $E$ that $F$ is open. Since $\Omega=E \cup F$, with $E \cap F=\varnothing$, and $\Omega$ is a connected set, then $E=\Omega$, in this case $K(f)=\Omega$, or $E=\varnothing$, in this case $K(f)$ does not have a point of accumulation in $\Omega$.

Remark 4.6. It should be observed that the result of Lemma 4.5 remains valid if we replace $K(f)$ by $K_{1}(f)=\{\lambda \in \Omega$ such that $f(\lambda)$ is weakly compact $\}$.

Lemma 4.7. If $O$ is an open and connected set of $\mathbb{C}$ and $F$ is a set of isolated points of $O$, then $O^{\prime}=O \backslash F$ is a connected set of $\mathbb{C}$.

Proposition 4.8. Let $R \in \mathcal{L}(X)$.

(i) If $\rho_{\mathrm{ess}}(T+R)$ is a connected set, then for all Kcompact operator, $\rho(T+R+K)$ is a connected set.

(ii) If $R$ is $T$-Regular, then for all $K$ compact operator, $\rho(T+R+K) \cap \rho(T+R) \cap \rho(T)$ has a point of accumulation.

(iii) If $R$ is $T$-Regular and $\rho(T+R)$ is a connected set of $\mathbb{C}$, then for all $\lambda \in \rho(T+R),[(\lambda-T-$ $\left.R)^{-1} R\right]^{4}$ is compact.

Proof. (i) For all $K$ compact operator, we have $\rho_{\text {ess }}(T+R)=\rho_{\text {ess }}(T+R+K)$. Since $\rho_{\text {ess }}(T+R)$ is a connected set, then from [20, Lemma 3.1],

$$
\mathbb{C} \backslash \rho_{6}(T+R+K)=\sigma_{\text {ess }}(T+R+K)=\sigma_{\text {ess }}(T+R),
$$


where $\rho_{6}(T+R+K)$ denotes the set of those $\lambda \in \rho_{\text {ess }}(T+R+K)$ such that all scalars near $\lambda$ are in $\rho(T+R+K)$. The result follows from the identity

$\rho(T+R+K)$

$=C_{\sigma_{e_{6}(T+R+K)}} \backslash\{\lambda \in \sigma(T+R+K) ; \lambda$ is an isolated eigenvalue of finite algebraic multiplicity $\}$

and Lemma 4.7.

(ii) It suffices to show that $\rho(T+R+K) \cap \rho(T+R) \cap \rho(T)$ is nonempty because for every open nonempty set, all of its points are points of accumulation. Since $T$ satisfies the hypothesis $(\mathcal{A})$, there exist $\lambda_{1}, \lambda_{2}$, and $\lambda_{3} \in \mathbb{R}$ such that $] \lambda_{1},+\infty[\subset \rho(T),] \lambda_{2},+\infty[$ $\subset \rho(T+R)$, and $] \lambda_{3},+\infty\left[\subset \rho(T+R+K)\right.$. If we take $\bar{\lambda}=\max \left\{\lambda_{1}, \lambda_{2}, \lambda_{3}\right\}$, we have necessarily ] $\bar{\lambda},+\infty[\subset \rho(T+R+K) \cap \rho(T+R) \cap \rho(T)$. Then the set $\rho(T+R+K) \cap \rho(T+R) \cap \rho(T)$ has a point of accumulation.

(iii) Let $E=\left\{\lambda \in \rho(T+R)\right.$ such that $\left[(\lambda-T-R)^{-1} R\right]^{4}$ is compact $\}$. From Lemma 4.3, we have $\rho(T+R) \cap \rho(T) \subset E$. Applying the assertion (ii), $\rho(T+R) \cap \rho(T)$ has a point of accumulation. Finally, by Lemma $4.5, E=\rho(T+R)$. This completes the proof of the proposition.

Lemma 4.9. Let $K$ be a compact operator and assume that $R$ is $T$-Regular. Then

(i) for all $\lambda \in \rho(T+K) \cap \rho(T), R(\lambda-T-K)^{-1} R$ is weakly compact,

(ii) for all $\lambda \in \rho(T+R+K)$, $\left[(\lambda-T-R-K)^{-1} R\right]^{4}$ is compact.

Proof. (i) By using the resolvent equation, we get the following identity:

$$
R(\lambda-T-K)^{-1} R=R(\lambda-T-K)^{-1} K(\lambda-T)^{-1} R+R(\lambda-T)^{-1} R
$$

Since $R(\lambda-T-K)^{-1} K(\lambda-T)^{-1} R$ is compact and $R(\lambda-T)^{-1} R$ is weakly compact, then $R(\lambda-T-$ $K)^{-1} R$ is weakly compact.

(ii) For $\lambda \in \rho(T+R+K) \cap \rho(T)$, we have

$$
(\lambda-T-R-K)^{-1} R=(\lambda-T)^{-1} R+(\lambda-T-R-K)^{-1}(R+K)(\lambda-T)^{-1} R=A_{1}+A_{2}+A_{3},
$$

where $A_{1}=(\lambda-T)^{-1} R, A_{2}=(\lambda-T-R-K)^{-1} R(\lambda-T)^{-1} R$, and $A_{3}=(\lambda-T-R-K)^{-1} K(\lambda-T)^{-1} R$. Hence

$$
\left[(\lambda-T-R-K)^{-1} R\right]^{4}=\left(A_{1}+A_{2}+A_{3}\right)^{4}=\sum_{j=1}^{3^{4}} Q_{j} .
$$

For each $j \in\left\{1, \ldots, 3^{4}\right\}$, the operator $Q_{j}$ is compact, so $\left[(\lambda-T-R-K)^{-1} R\right]^{4}$ is compact. Let $E^{\prime}=\left\{\lambda \in \rho(T+R+K)\right.$ such that $\left[(\lambda-T-R-K)^{-1} R\right]^{4}$ is compact $\}$. We have $\rho(T+R+K) \cap \rho(T) \subset E^{\prime}$. By Proposition 4.8(ii), $E^{\prime}$ has a point of accumulation in $\rho(T+R+K)$. By Proposition 4.8(i), $\rho(T+R+K)$ is a connected set. Finally, by Lemma $4.5, E^{\prime}=\rho(T+R+K)$. 
Lemma 4.10. Assume that $R$ is $T$-Regular. Let $\partial_{T}(X)$ be a subgroup of $(\mathcal{\perp}(X),+)$ such that $\partial_{T}(X) \subset$ $\mathcal{R}_{T}(X)$ and let

$$
\supset_{T}(X)=\left\{R+K \in \mathcal{L}(X) \text {, such that } K \text { is compact and } R \in \partial_{T}(X)\right\} \text {. }
$$

Then

(i) $\mathcal{K}(X) \subset \supset_{T}(X) \subset \mathcal{G}_{T}^{4}(X)$,

(ii) for all $\left(R_{1}+K_{1}\right),\left(R_{2}+K_{2}\right) \in \supset_{T}(X)$, then $\left(R_{1}+K_{1}\right) \pm\left(R_{2}+K_{2}\right) \in \supset_{T}(X)$.

Proof. (i) Since the null operator $\tilde{o} \in \partial_{T}(X)$, then $\mathcal{K}(X) \subset \supset_{T}(X)$.

Let $R+K \in \supset_{T}(X)$ and let $\lambda \in \rho(T+R+K)$. We have

$$
\left[(\lambda-T-R-K)^{-1}(R+K)\right]^{4}=\left[(\lambda-T-R-K)^{-1} R+(\lambda-T-R-K)^{-1} K\right]^{4}=\sum_{j=1}^{2^{4}} P_{j}
$$

where each $P_{j}$ is a product of 4 factors formed from the operators $(\lambda-T-R-K)^{-1} R$ and $(\lambda-T-R-K)^{-1} K$. From Lemma $4.9, P_{1}=\left[(\lambda-T-R-K)^{-1} R\right]^{4}$ is compact. For $j \in\left\{2, \ldots, 2^{4}\right\}$, the operator $K$ appears at least one time in the expression of $P_{j}$. So $P_{j}$ is compact. Hence $\left[(\lambda-T-R-K)^{-1}(R+K)\right]^{4}$ is compact for all $\lambda \in \rho(T+R+K)$.

(ii) It is clear that for all $\left(R_{1}+K_{1}\right),\left(R_{2}+K_{2}\right) \in \supset_{T}(X)$, we have $\left(R_{1}+K_{1}\right) \pm\left(R_{2}+K_{2}\right)=$ $\left(R_{1} \pm R_{2}\right)+\left(K_{1} \pm K_{2}\right) \in \supset_{T}(X)$.

We are now ready to prove the main result of this section.

Theorem 4.11. Let $\partial_{T}(X)$ be a subgroup of $(\mathcal{L}(X),+)$ such that $\partial_{T}(X) \subset \mathcal{R}_{T}(X)$. Then for all $R \in$ $\partial_{T}(X)$

$$
\sigma_{\mathrm{ess}}(T+R)=\sigma_{\mathrm{ess}}(T)
$$

Proof. The result follows from Lemma 4.10 and Corollary 3.7.

Corollary 4.12. Let $R \in \mathcal{L}(X)$ such that for all $n \in \mathbb{Z}, n R$ is $T$-regular. Then

$$
\sigma_{\text {ess }}(T+R)=\sigma_{\text {ess }}(T) \text {. }
$$

Proof. Let $\partial_{T}(X)=\{n R, n \in \mathbb{Z}\}$. We have $\partial_{T}(X) \subset R_{T}(X)$ and for all $R_{1}, R_{2} \in \partial_{T}(X)$, $R_{1} \pm R_{2} \in \partial_{T}(X)$. Then by Theorem 4.11, we have $\sigma_{\text {ess }}(T+R)=\sigma_{\text {ess }}(T)$.

\section{Application to transport equation}

In this section, we will apply the result of Theorem 4.11 to investigate the Scheter essential spectrum to the multidimensional neutron transport equation which governs the time evolution of the distribution of neutrons in a nuclear reactor (cf. [7, 14, 22, 26, 32]):

$$
\begin{gathered}
\frac{\partial \psi}{\partial t}(x, v, t)=-v \frac{\partial \psi}{\partial x}(x, v, t)-\sigma(v) \psi(x, v, t)+\int_{V} k\left(x, v, v^{\prime}\right) \psi\left(x, v^{\prime}, t\right) d v^{\prime} \\
=A_{0} \psi(x, v, t)=T_{0} \psi(x, v, t)+R \psi(x, v, t) \\
\psi_{\mid \Gamma_{-}}=0, \quad \psi(x, v, 0)=\psi_{0}(x, v)
\end{gathered}
$$


where $T_{0}$ is the streaming operator and $R$ denotes the integral part of $A_{0}$ (the collision operator), $(x, v) \in D \times V$, where $D=\stackrel{\circ}{D} \subset \mathbb{R}^{N}$ and the velocity space $V \subset \mathbb{R}^{N}(N \geq 1)$. The unbounded operator $A_{0}$ is studied in the Banach space $X_{1}=L_{1}(D \times V, d x d v)$. Its domain is

$$
\Phi\left(A_{0}\right)=\boldsymbol{\Phi}\left(T_{0}\right)=\left\{\psi \in X_{1} \text {, such that } v \frac{\partial \psi}{\partial x} \in X_{1}, \psi_{\mid \Gamma_{-}}=0\right\}
$$

where

$$
\Gamma_{-}=\{(x, v) \in \partial D \times V \text { such that } v \text { is ingoing at } x \in \partial D\} .
$$

The function $\sigma(\cdot)$ is called the collision frequency. The scattering kernel $\kappa(\cdot, \cdot, \cdot)$ defines a linear operator $R$ by

$$
R: X_{1} \longrightarrow X_{1}, \quad \psi \longrightarrow \int_{V} k\left(x, v, v^{\prime}\right) \psi\left(x, v^{\prime}\right) d v^{\prime}
$$

Observe that the operator $R$ acts only on the variables $v^{\prime}$. So, $x$ may be viewed merely as a parameter in $D$. Hence, we may consider $R$ as a function

$$
R(\cdot): x \in D \longrightarrow R(x) \in Z,
$$

where $Z=\mathcal{L}\left(L_{1}(V, d v)\right)$ denotes the set of all bounded linear operators on $L_{1}(V, d v)$. In the following we will make the assumptions (hypothesis 41 ):

(i) the function $R(\cdot)$ is strongly measurable,

(ii) there exists a compact subset $\mathcal{C} \subset \mathcal{L}\left(L_{1}(V, d v)\right)$ such that $R(x) \in \mathcal{C}$ a.e. on $D$,

(iii) $R(x) \in \mathcal{K}\left(L_{1}(V, d v)\right)$ a.e. on $D$,

where $\mathcal{K}\left(L_{1}(V, d v)\right)$ denotes the set of all compact operators on $L_{1}(V ; d v)$.

Obviously, the second hypothesis of $\mathscr{A 1}$ implies that

$$
R(\cdot) \in L^{\infty}(D, Z)
$$

Let $\psi \in X_{1}$. It is easy to see that $(R \psi)(x, v)=R(x) \psi(x, v)$ and then, by $\mathcal{A} 1$, we have

$$
\int_{V}|(R \psi)(x, v)| d v \leq\|R(\cdot)\|_{L^{\infty}(D, Z)} \int_{V}|\psi(x, v)| d v,
$$

and therefore,

$$
\int_{D} \int_{V}|(R \psi)(x, v)| d v \leq\|R(\cdot)\|_{L^{\infty}(D, Z)} \int_{D} \int_{V}|\psi(x, v)| d v .
$$

Thus leads to the estimate

$$
\|R\|_{\mathcal{L}\left(X_{1}\right)} \leq\|R(\cdot)\|_{L^{\infty}(D, Z)} .
$$

Definition 5.1. A collision operator $R$ is said to be regular if it satisfies the assumption $A 1$ above.

We denote by $\mathcal{R}\left(X_{1}\right)$ the space of all regular operator. 
It is well known that

$$
\sigma\left(T_{0}\right)=\left\{\lambda \in \mathbb{C} \text { such that } \operatorname{Re} \lambda \leq-\lambda^{*}\right\}, \quad \text { where } \lambda^{*}:=\liminf _{\xi \mid \rightarrow 0} \sigma(\xi) .
$$

(see, e.g., [22, Corollary 12.11, page 272]). Note that the spectrum of the operator $T_{0}$ was analyzed in [28]. In particular, we have

$$
\sigma_{\text {ess }}\left(T_{0}\right)=\sigma C\left(T_{0}\right)=\left\{\lambda \in \mathbb{C} \text { such that } \operatorname{Re} \lambda \leq-\lambda^{*}\right\},
$$

where $\sigma C\left(T_{0}\right)$ denotes the continuous spectrum of $T_{0}$. The existence of the eigenvalues of $T_{0}+R$ in the half-plan $\left\{\lambda \in \mathbb{C}\right.$ such that $\left.\operatorname{Re} \lambda>-\lambda^{*}\right\}$ is related to the compactness of some iterate of $\left(\lambda-T_{0}\right)^{-1} R$ (see [22, Chapter 12]).

Lemma 5.2 (see [28, Lemma 2.1]). Let $K$ and $H$ be two regular collision operators on $X_{1}$ and $\operatorname{Re} \lambda>$ $\eta$, where $\eta$ is the type of the $C_{0}$-semigroup generated by $T_{0}$.

(i) $K\left(\lambda-T_{0}\right)^{-1} H$ is weakly compact on $X_{1}$. If $\sigma(x, v)=\sigma(v)$ and if $D$ is convex, then $K\left(\lambda-T_{0}\right)^{-1} H$ is compact on $X_{1}$.

(ii) If $\omega>\eta$, then $\lim _{|\operatorname{Im} \lambda| \rightarrow+\infty}\left\|K\left(\lambda-T_{0}\right)^{-1} H\right\|=0$ uniformly in $\{\lambda ; \operatorname{Re} \lambda \geq \omega\}$.

Theorem 5.3. Let $R$ be a regular operator such that, for all $n \in \mathbb{Z}, \rho_{\mathrm{ess}}\left(T_{0}+n R\right)$ is a connected set of C. Then

$$
\sigma_{\text {ess }}\left(T_{0}+R\right)=\sigma_{\text {ess }}\left(T_{0}\right)=\left\{\lambda \in \mathbb{C} \text { such that } \operatorname{Re} \lambda \leq-\lambda^{*}\right\} .
$$

Proof. We claim that, for all $n \in \mathbb{Z}, n R$ is $T_{0}$-regular. Indeed, we have that $n R$ is regular and so, by Lemma $5.2, n R\left(\lambda-T_{0}\right)^{-1} n R$ is weakly compact on $X_{1}$, for all $\lambda$ such that $\operatorname{Re}(\lambda)>\eta$. The use of Remark 4.6 and the fact that $\rho\left(T_{0}\right)$ is a connected set of $\mathbb{C}$ shows that $n R\left(\lambda-T_{0}\right)^{-1} n R$ is weakly compact for all $\lambda \in \rho\left(T_{0}\right)$. So, for all $n \in \mathbb{Z}, n R$ is $T_{0}$-regular. We define $\partial_{T_{0}}\left(X_{1}\right)=$ $\{n R, n \in \mathbb{Z}\}$. We have $\partial_{T_{0}}\left(X_{1}\right) \subset \mathcal{R}_{T_{0}}\left(X_{1}\right)$ and for all $R_{1}, R_{2} \in \partial_{T_{0}}\left(X_{1}\right)$, we have $R_{1} \pm R_{2} \in \partial_{T_{0}}\left(X_{1}\right)$. Finally, by Theorem 4.11, we obtain $\sigma_{\text {ess }}\left(T_{0}+R\right)=\sigma_{\text {ess }}\left(T_{0}\right)=\left\{\lambda \in \mathbb{C}\right.$ such that $\left.\operatorname{Re} \lambda \leq-\lambda^{*}\right\}$. This completes the proof of the theorem.

\section{References}

[1] R. R. Akhmerov, M. I. Kamenskiǔ, A. S. Potapov, A. E. Rodkina, and B. N. Sadovskiǔ, Measures of Noncompactness and Condensing Operators, vol. 55 of Operator Theory: Advances and Applications, Birkhäuser, Basel, Switzerland, 1992.

[2] K. Astala, "On measures of noncompactness and ideal variations in Banach spaces," Annales Academiae Scientiarum Fennicae. Series A I. Mathematica Dissertationes, no. 29, p. 42, 1980.

[3] J. Banaś and K. Geobel, "Measures of noncompactness in Banach spaces," in In Lecture Notes in Pure and Applied Mathematics, vol. 60, pp. 259-262, Marcel Dekker, New York, NY, USA, 1980.

[4] J. Daneš, "On the Istratescu measure of noncompactness," Bulletin Mathématique de la Société des Sciences Mathématiques de la République Socialiste de Roumanie, vol. 16, no. 64, pp. 403-406, 1972.

[5] J. Diestel, "A survey of results related to the Dunford-Pettis property," in Proceedings of the Conference on Integration, Topology, and Geometry in Linear Spaces, vol. 2 of Contemp. Math., pp. 15-60, American Mathematical Society, Chapel Hill, NC, USA, 1980.

[6] T. Domínguez Benavides, "Some properties of the set and ball measures of noncompactness and applications," Journal of the London Mathematical Society, vol. 34, no. 1, pp. 120-128, 1986.

[7] J. J. Duderstart and W. R. Martin, Transport Theory, John Wiley \& Sons, New York, NY, USA, 1979. 
[8] N. Dunford and J. T. Schwartz, Linear Operators. Part 1, Interscience, New York, NY, USA, 1958.

[9] N. Dunford and B. J. Pettis, "Linear operations on summable functions," Transactions of the American Mathematical Society, vol. 47, no. 3, pp. 323-392, 1940.

[10] D. E. Edmum and W. D. Evans, Spectral Theory and Differential Operators, Oxford Science Publications, The Clarendon Press, Oxford University Press, New York, NY, USA, 1987.

[11] I. C. Gohberg, A. S. Marku, and I. A. Feldman, "Normally solvable operators and ideals associated with them," Journal of the American Mathematical Society, vol. 61, pp. 63-84, 1967.

[12] S. Goldbert, Unbounded Linear Operators, McGraw-Hill, New York, NY, USA, 1966.

[13] B. Gramsch and D. Lay, "Spectral mapping theorems for essential spectra," Mathematische Annalen, vol. 192, no. 1, pp. 17-32, 1971.

[14] W. Greenberg, C. van der Mee, and V. Protopopescu, Boundary Value Problems in Abstract Kinetic Theory, vol. 23 of Operator Theory: Advances and Applications, Birkhäuser, Basel, Switzerland, 1987.

[15] A. Grothendieck, "Sur les applications linéaires faiblement compactes d'espaces du type $C(K)$," Canadian Journal of Mathematics, vol. 5, pp. 129-173, 1953.

[16] K. Gustafson and J. Weidmann, "On the essential spectrum," Journal of Mathematical Analysis and Applications, vol. 25, no. 1, pp. 121-127, 1969.

[17] A. Jeribi, "Une nouvelle caractérisation du spectre essentiel et application," Comptes Rendus de l'Académie des Sciences, vol. 331, no. 7, pp. 525-530, 2000.

[18] A. Jeribi, "A characterization of the essential spectrum and applications," Bollettino della Unione Matematica Italiana. Serie VIII. Sezione B, vol. 5, no. 3, pp. 805-825, 2002.

[19] A. Jeribi, "A characterization of the Schechter essential spectrum on Banach spaces and applications," Journal of Mathematical Analysis and Applications, vol. 271, no. 2, pp. 343-358, 2002.

[20] A. Jeribi and M. Mnif, "Fredholm operators, essential spectra and application to transport equations," Acta Applicandae Mathematicae, vol. 89, no. 1-3, pp. 155-176, 2005.

[21] M. A. Kaashoek and D. C. Lay, "Ascent, descent, and commuting perturbations," Transactions of the American Mathematical Society, vol. 169, pp. 35-47, 1972.

[22] H. G. Kaper, C. G. Lekkerkerker, and J. Hejtmanek, Spectral Methods in Linear Transport Theory, vol. 5 of Operator Theory: Advances and Applications, Birkhäuser, Basel, Switzerland, 1982.

[23] K. Latrach, "Essential spectra on spaces with the Dunford-Pettis property," Journal of Mathematical Analysis and Applications, vol. 233, no. 2, pp. 607-622, 1999.

[24] K. Latrach and A. Dehici, "Relatively strictly singular perturbations, essential spectra, and application to transport operators," Journal of Mathematical Analysis and Applications, vol. 252, no. 2, pp. 767-789, 2000.

[25] K. Latrach and A. Jeribi, "Some results on Fredholm operators, essential spectra, and application," Journal of Mathematical Analysis and Applications, vol. 225, no. 2, pp. 461-485, 1998.

[26] K. Latrach and J. M. Paoli, "Polynomially compact-like strongly continuous semigroups," Acta Applicandae Mathematicae, vol. 82, no. 1, pp. 87-99, 2004.

[27] M. M. Milovanović-Arandjelović, "Measures of noncompactness on uniform spaces-the axiomatic approach," Filomat, no. 15, pp. 221-225, 2001.

[28] M. Mokhtar-Kharroubi, "Time asymptotic behaviour and compactness in transport theory," European Journal of Mechanics B, vol. 11, no. 1, pp. 39-68, 1992.

[29] V. Rakočević, "Measures of noncompactness and some applications," Filomat, no. 12, part 2, pp. 87120, 1998.

[30] M. Schechter, Principles of Functional Analysis, Academic Press, New York, NY, USA, 1971.

[31] M. Schechter, "On the essential spectrum of an arbitrary operator," Journal of Mathematical Analysis and Applications, vol. 13, pp. 204-215, 1965.

[32] M. Wing, An Introduction to Transport Theory, John Wiley \& Sons, New York, NY, USA, 1962. 